\title{
A Fast and Efficient Technique to Simulate the Effects of Geomagnetic Storms Recorded by GRAPES-3 in Real-Time
}

M. Zuberi ${ }^{* a b}$, S. Ahmad ${ }^{a c}$, A. Chandra ${ }^{a b}$, S.R. Dugad ${ }^{a b}$, S.K. Gupta ${ }^{a b}$, B. Hariharan ${ }^{a b}$, Y. Hayashi ${ }^{a d}$, P. Jagadeesan ${ }^{a b}$, A. Jain ${ }^{a b}$, V.B. Jhansi ${ }^{a b}$, S. Kawakami ${ }^{a d}$, H. Kojima $^{a e}$, P.K. Mohanty ${ }^{a b}$, S.D. Morris ${ }^{a b}$, P.K. Nayak ${ }^{a b}$, A. Oshima ${ }^{a f}$, P.S. Rakshe ${ }^{a b}$, K. Ramesh ${ }^{a b}$, B.S. Rao ${ }^{a b}$, L.V. Reddy ${ }^{a b}$, S. Shibata ${ }^{a f}$

${ }^{a}$ The GRAPES-3 Experiment, Cosmic Ray Laboratory, Raj Bhavan, Ooty 643001, India

${ }^{b}$ Tata Institute of Fundamental Research, Mumbai 400005, India

${ }^{c}$ Aligarh Muslim Univeristy, Aligarh 202002, India

${ }^{d}$ Graduate School of Science, Osaka City University, Osaka 558-8585, Japan

${ }^{e}$ Faculty of Engineering, Aichi Insitute of Technology, Toyota City, Aichi 470-0392, Japan

${ }^{f}$ College of Engineering, Chubu University, Kasugai, Aichi 487-8501, Japan

E-mail: meeran.zuberihep@gmail.com

Following the GRAPES-3 discovery of a transient weakening of Earth's magnetic shield through observation of a cosmic ray burst on 22 June 2015, we have been involved in an effort to search for more such events in 20 years of archived data. An important step in analyzing the data is to simulate the cosmic ray trajectories in the geomagnetic field influenced by the interplanetary magnetic field during a geomagnetic storm. The simulation of 22 June 2015 burst required over two months of running time on the 1280 core GRAPES-3 computer cluster in Ooty. We have developed a fast, efficient technique which has allowed us to simulate the 22 June 2015 event in a fraction of a second with the same resolution as the earlier computational-intensive method. This has laid the foundation for quick analysis of a large number of such events in archival data, and of future events virtually in real-time. Details of this method will be presented during the conference.

36th International Cosmic Ray Conference -ICRC2019-

July 24th - August 1st, 2019

Madison, WI, U.S.A.

* Speaker. 


\section{Introduction}

Geomagnetic storms (GMS) are major disturbances in the Earth magnetosphere due to change in interplanetary magnetic field (IMF) towards southward direction and remain so for a long time. Reconnection between the southward directed IMF component with the northward directed Earth geomagnetic field (GMF) allow solar wind energy transport into the Earth magnetosphere $[1,2]$. The GMS is well associated with Earth directed coronal mass ejections (CMEs) which are eruption of solar magnetic field and plasma occurs due to large-scale magnetic rearrangements in the solar atmosphere [3, 4]. These CMEs can have large societal impact by triggering severe storms that disrupt space and ground based communications. There have been many advances in the field of GMS forecasting by understanding their properties in past many decades. However, the accurate predictions of such storms is yet to established.

\section{Conventional Simulation}

On 22 June 2015, GRAPES-3 muon telescope (G3MT) observed a 'muon burst' which started at 19:00 UT and lasted for two hours riding on a large Forbush decrease (minimum change of $2.5 \%$ ) and solar diurnal anisotropy variations. By using Fast Fourier Transform (FFT) analysis, this event was studied in details which is described elsewhere [5, 6, 7]. This phenomena is based on the assumption of lowering the cutoff rigidity $\left(\mathrm{R}_{C}\right)$, allowed more low energy cosmic rays to enter Earth and results the excess of muon flux which was observed in all nine directions simultaneously. To test this hypothesis, a certain strategy was followed as shown by block diagram in Fig. 1.

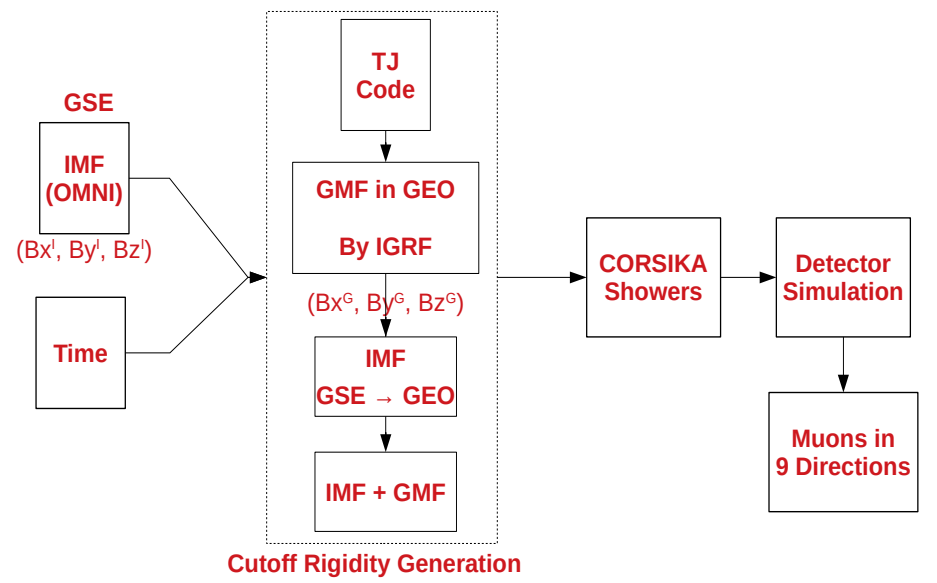

Figure 1: Conventional Simulation flow chart

Firstly, muon telescope field of view was divided in the fine spatial resolution of $360^{\circ} \times 60^{\circ}$ grids, each $1^{o}$ in azimuth and zenith directions. After transforming IMF data components $\left(\mathrm{B}_{x}, \mathrm{~B}_{y}\right.$ and $\mathrm{B}_{z}$ averaged every 4 mins) obtained from OMNI database [8] from Geocentric Solar Ecliptic (GSE) coordinate system to Geographic (GEO) coordinate system, they are vectorially added to the geomagnetic field components (GMF) components calculated from the IGRF-11 model [9]. For 
every value of IMF perturbed GMF, $\mathrm{R}_{C}$ was calculated by using back-tracing method written by D. F. Smart and M. A. Shea [10]. This method calculates $\mathrm{R}_{C}$ by launching an anti-proton started with higher rigidity from observer's location to space. The anti-proton is checked for GMF with a decreasing rigidity from the initial value. The $\mathrm{R}_{C}$ is obtained from this method the rigidity at which the anti-proton escapes from the GMF vicinity to space. This process is repeated for each $1^{o}$ grid of zenith and azimuthal angles to get rigidity map. By using this map and detector simulations the CORSIKA [11] muons are reconstructed into 9 directions by satisfying the trigger requirements of G3MT. For the baseline unperturbed GMF is used to get the muons in nine directions and for IMF calculated for every four minute interval. The nine simulated profiles showed very high correlations of $-0.89 \pm 0.05$ between the observed and simulated 'muon burst' which is described elsewhere $[5,6,7]$. The above mentioned process require large scale computing as it took $\sim 2$ months of computation time by using 1280 core GRAPES-3 computer cluster.

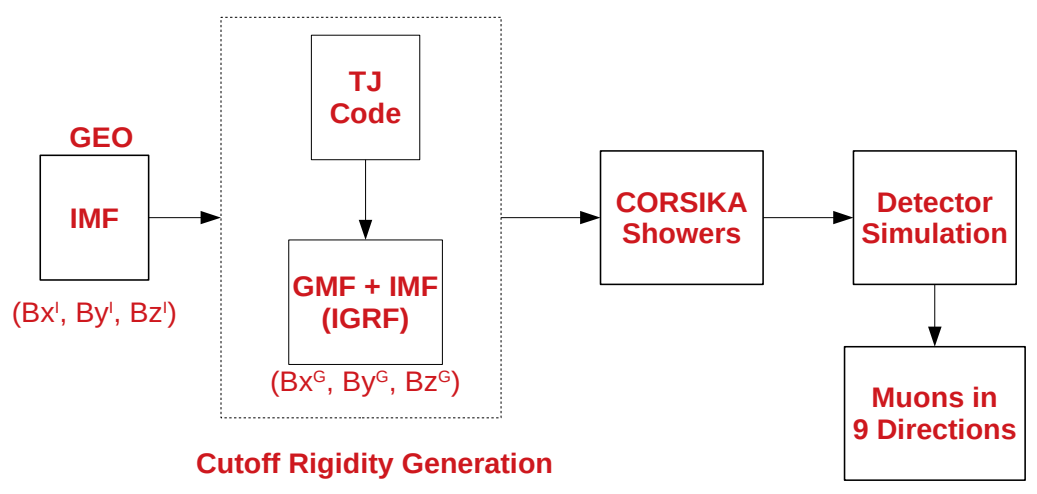

Figure 2: Time Independent Simulation flow chart

The IMF information comes from the satellite located at first Lagrangian point (L1) which is $\sim 1.5$ million km away from the Earth in the Earth-Sun line. However, we do not know how these information changes after crossing L1. For ex. in case of above mentioned event, the GMS suppose to reach Earth within $\sim 35$ mins based on the available information from satellite, but, this event took additional 28 mins delay shown by our analysis. In future if there will be any severe GMS comes, the only possibility will be to do a complete power shut down for prevention of any damage from it. But, if we only relay on satellite based warnings, it may cost a huge financial loss for every min of unnecessary power shutdown. However, this problem can tackled by providing additional inputs of GMS arrival time from ground based detectors such as GRAPES-3. But to device such mechanism, the properties of 'burst-like' events should be well understood by analyzing more of them. During solar active period, 50 G3-G5 class storms occurs in a year. In 20 years of GRAPES-3 muons archival data, we can expect many of such events. But the speed of simulation ( $\sim 1$ month for one event) makes this task almost impossible to analyze large number of events. 
This emerges a need of an alternative approach to do time efficient simulations so that one can quickly analyze all available archival data and lead to a definitive mechanism for GMS forecasting.

\section{New Simulation}

The conventional simulation uses the IMF data in GSE coordinate system, which later transformed into GEO coordinates by superposing on dipolar geomagnetic field in calculations of $\mathrm{R}_{C}$ for a given time. Due to Earth orbital motion and its rotation, the simulated values becomes different for different time in a year, depending on how IMF individual components getting resolved after transformations. In the new method we have divided this simulation into time independent and time dependent parts as discussed in the following sub sections.

\subsection{Time Independent Part}

Since the time dependency of the simulation comes from the transformations of IMF from GSE to GEO coordinate system. Instead of transforming IMF values, the individual IMF components were used in GEO coordinate system itself for $\mathrm{R}_{C}$ calculations and that were further used for detector simulation by using CORSIKA generated showers as described in Fig. 2.

The effect of individual IMF components were simulated for the range of -50 $\mathrm{nT}$ to $50 \mathrm{nT}$ in steps of $5 \mathrm{nT}$ for all nine directions. In case of vertical direction, a large negative dependence of $-0.002 \% n T^{-1}$ were observed for $\mathrm{B}_{Z}$ by using liner fit. However, $\mathrm{B}_{Y}$ and $\mathrm{B}_{X}$ showed very weak positive dependence of $0.0003 \% n T^{-1}$ and $0.00003 \% n T^{-1}$ respectively as shown in Fig. 3. The effect of $\mathrm{B}_{X}$ can be safely neglected due its least magnitude which is smaller then the resolution of muon data. The similar dependence of individual IMF components were observed in other directions also. Similarly, the fitting parameters were calculated for all nine directions which describes the effect of GMF perturbed by IMF in GEO coordinate system for any given

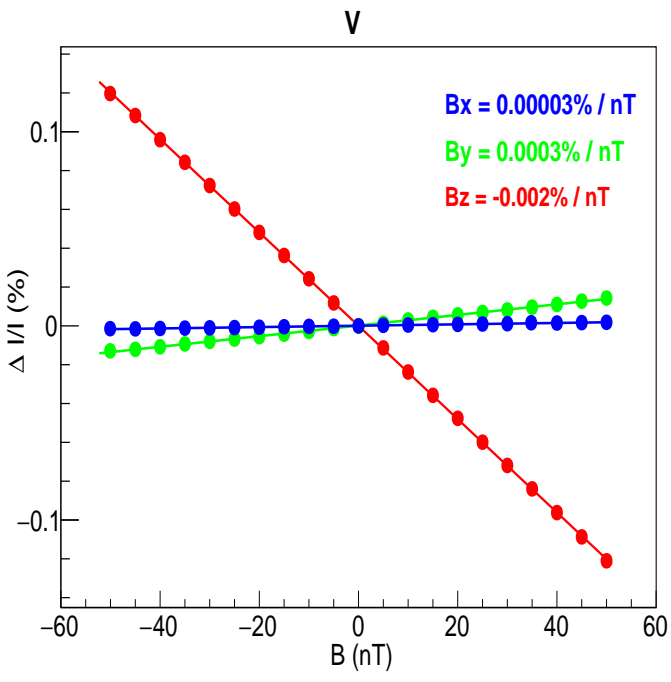

Figure 3: $\mathrm{B}_{X}, \mathrm{~B}_{Y}$ and $\mathrm{B}_{Z}$ dependence for vertical direction time.

The additive property of individual IMF components were also checked by taking the ratio between individual components effect sum (by keeping the other two zero) to the effect when all components were present simultaneously. The ratio comes $\sim 1$ at different field values for all nine directions, which clearly shows its additive property as shown in Fig. 4 for vertical direction. 


\subsection{Time Dependent Part}

After parameterizing the effect of individual field components in GEO coordinates, the IMF can be transformed from GSE to GEO coordinates [12] as:

1) First, IMF was transformed from GSE to Geocentric Equatorial Inertial (GEI) coordinate System by performing the rotation in the plane of the ecliptic from the Earth-Sun direction to the first point of Aries and then by doing the another rotation plane of the ecliptic to the Earth's equator, can be expressed as

$$
T_{1}=\left(<\lambda_{\odot}, Z>\times<\varepsilon, X>\right)^{-1}
$$

where, the two angles are calculated as follows 'U.S. Naval Observatory, 1989'. First, the obliquity of the ecliptic, $\varepsilon$ as

$$
\varepsilon=23.439-0.013 \times T_{0}
$$

and then, the Sun's ecliptic longitude $\left(\lambda_{\odot}\right)$ as

$$
\begin{gathered}
M=357.528+35999.050 \times T_{0}+0.04107 U T \\
\Lambda=280.460+36000.772 \times T_{0}+0.04107 U T \\
\lambda_{\odot}=\Lambda+\left(1.915-0.0048 \times T_{0}\right) \times \sin (M)+0.020 \sin (2 M)
\end{gathered}
$$

where, $\mathrm{M}$ is the Sun's mean anomaly, $\Lambda$ its mean longitude and $T_{0}$ defined as

$$
T_{0}=\frac{M J D \times 51544.5}{36525.0}
$$

where, MJD is the modified Julian date ie time measured in fractional days from 00:00 UT on November 17, 1858.

2) Afterwards, from GEI coordinates, IMF was transformed to GEO by doing one more rotation in the plane of the Earth's geographic equator from the first point of Aries to Greenwich meridian described as

$$
T_{2}=<\theta, Z>
$$

where, the rotation angle $\theta$ is the Greenwich mean sidereal time which can be calculated

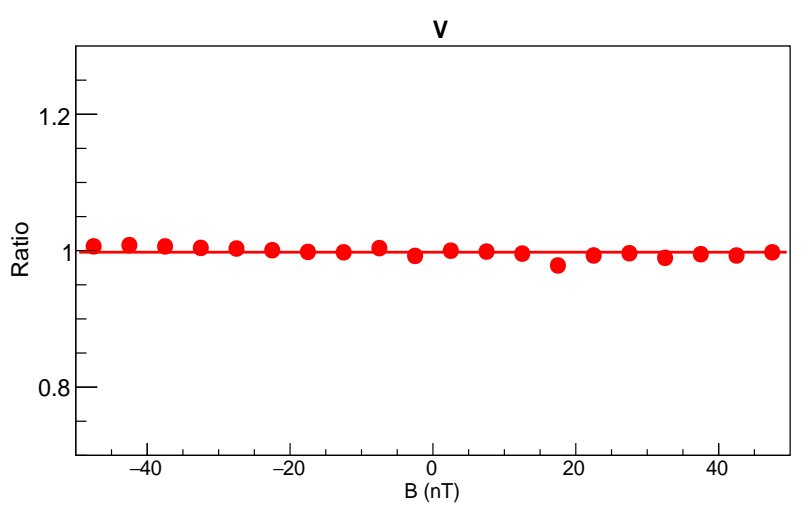

Figure 4: Ratios of field values for vertical dir 


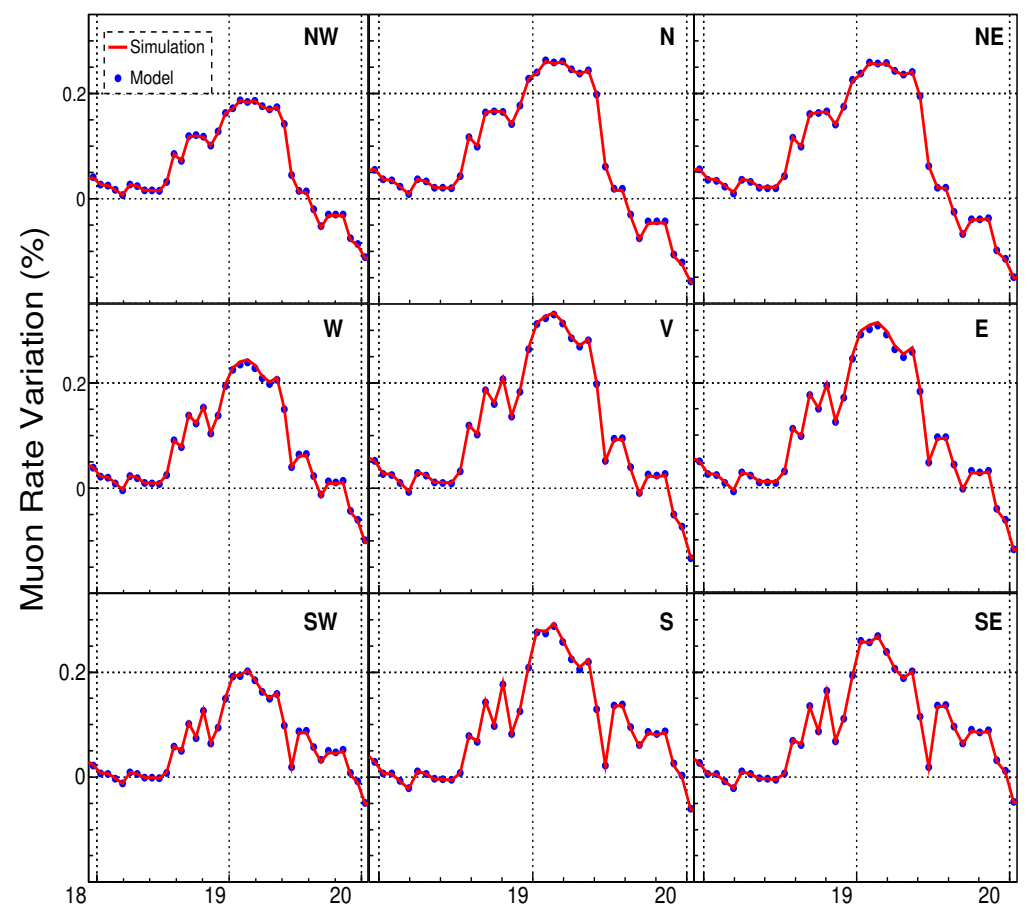

Figure 5: comparison between conventional and new simulations

using the following formula

$$
\theta=100.461+36000.770 \times T_{0}+15.04107 U T
$$

Now, the effect of GEO transformed IMF can be calculated by using liner regression equation as

$$
\begin{gathered}
\frac{\Delta I_{\alpha}}{I}(\%)=\left(\text { Slope }_{\alpha}\right) \times \alpha+\text { Intercept }_{\alpha} \\
\frac{\Delta I_{\text {Total }}}{I}(\%)=\Sigma \frac{\Delta I_{\alpha}}{I}(\%)
\end{gathered}
$$

where, $\alpha$ is $B_{X}, B_{Y}$ or $B_{Z}$ in GEO coordinate system, Slope $\alpha$ and Intercept $\alpha$ was calculated as explained in section 3.1.

\section{Results and Discussions}

The processing time limitation of conventional simulation for GMS effects on atmospheric muons arises a need of alternative method to do the same simulations quickly without producing 
any differences in final outputs. Unlike conventional simulation where the coordinate transformations of IMF were being done before simulation itself, in new method, the simulation was divided into two parts namely, time independent and time dependent parts. In time independent part, the $\mathrm{R}_{C}$ calculations and detector simulations were done for individual IMF components in GEO coordinate system itself. As shown in Fig. 3, for all three IMF components \% muons shows linear changes for different field values, which is due to the fact that the GMF value at Ooty is $\sim 43000$ $\mathrm{nT}$ and the magnitude of IMF which was superposed is maximum $\sim$ hundreds of $\mathrm{nT}$ which is much smaller then GMF and act as perturbation and can be expressed by Taylar expansion and the higher order terms can be neglected. Among all three components, $\mathrm{B}_{x}$ shows the least effect because it is in the direction of plasma. Since $\mathrm{B}_{Z}$ is perpendicular to the GMS, it shows maximum effect. In time dependent part, the IMF components was transformed from GSE to GEO coordinate system.

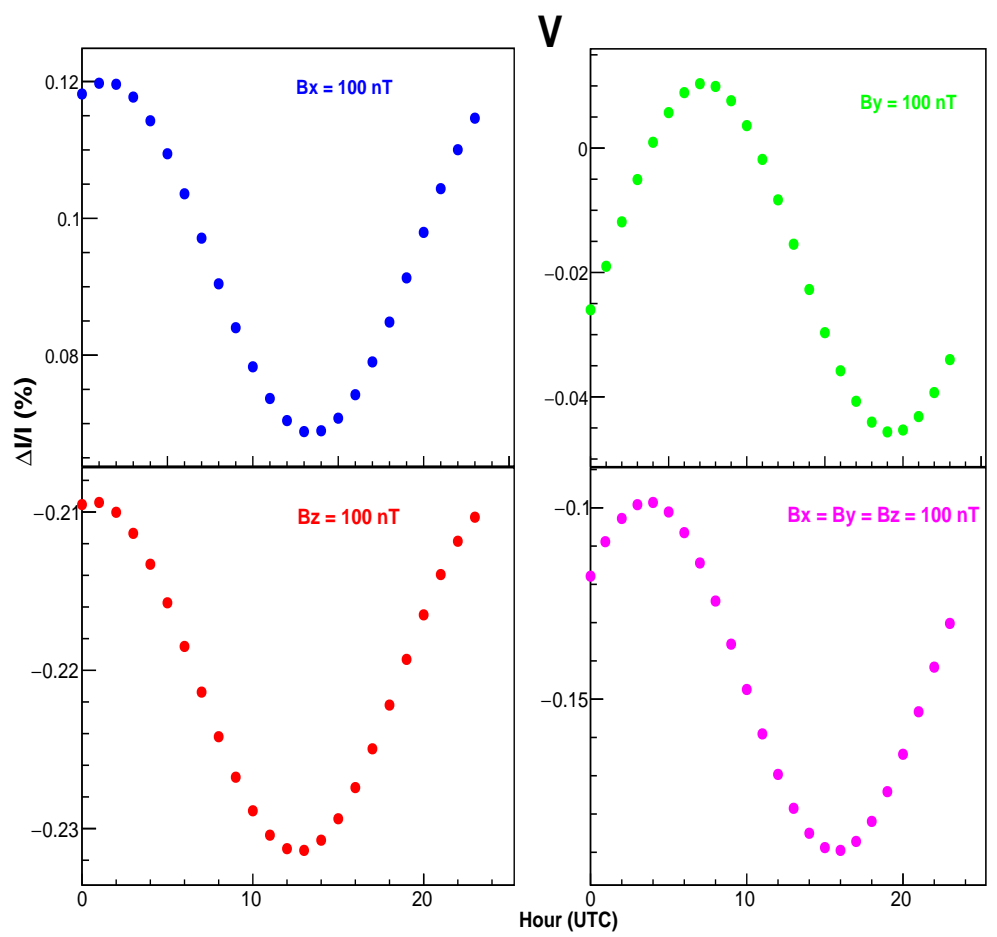

Figure 6: Daily variation of \% muons for individual and combined IMF components

For 22 June, 2015 muon-burst event, the results of conventional simulation and new simulation for all nine directions were compared. Fig. 5 shows the comparison between conventional and new simulations which is identical and the time taken by new simulation, which was the most critical parameter, was less than a second to produce this output. Since the simulation time becomes negligible due to implementation of new technique, daily and monthly effects were also studied for individual as well as combined IMF components. Fig. 6 shows the variation of percentage muons in vertical direction for 1 Jan 2015 at 100 nT IMF individual as well as combined components, which clearly shows the time dependency of observed effect. The monthly variations were studied by simulating the effect for every month first day at 0th hour. It shows that the observed percentage muons change is different in different months and the maximum effect were observed in May and 
June.

\section{Conclusions}

The discovery of muons burst on 22 June 2015 by GRAPES- 3 and the 28 mins delay for this particular event opens the possibilities of GMS forecasting by studying the properties of many such events. However, the requirement of intense computing resources for simulation of such events impose a big hurdle in it. A new simulation method was developed by studying the effects of individual IMF field components on muons and then parameterizing it. The time taken for simulation of 22 June 2015 event by using all available computational resources through conventional way was $\sim 2$ month which now can be done in less than a second by using new method. The advantage given by new method in terms of simulation time saving allows to analyze the archival data and analyze more burst-like events. Also this technique may give the possibility of GMS forecasting.

\section{Acknowledgement}

We thank D.B. Arjunan, G.P. Francis, V. Jeyakumar, S. Kingston, K. Manjunath, S. Murugapandian, S. Pandurangan, B. Rajesh, K. Ramadass, V. Santoshkumar, M.S. Shareef, C. Shobana, R. Sureshkumar and other colleagues for their help in running and maintenance of the GRAPES-3 experiment.

\section{References}

[1] Gonzalez, W. D., Tsurutani, B. T., Planet. Space Sci., 35, 1101 (1987).

[2] Tsurutani B. T., in Space Storms and Space Weather Hazard, I. A. Daglised (2001).

[3] N.Gopalswamy, Space Science Reviews, 124, 145âĂ ̌̌168 (2006).

[4] N.U.Crooker and T.S.Horbury, SpaceScienceReviews, 123, 93 âĂ ̌̌109 (2006).

[5] P.K. Mohanty, et al., Astroparticle Physics, 79, 23 (2016).

[6] P.K. Mohanty, et al., Phys. Rev. L, 117, 171101 (2016).

[7] P.K. Mohanty, et al., Phys. Rev. D, 97, 082001 (2018).

[8] http://omniweb.gsfc.nasa.gov/form.

[9] C. Finlayet al., Geophys. J. Int.183, 1216 (2010).

[10] D. F. Smart and M. A. Shea, Adv. Space Res, 36, 2012 (2005).

[11] https://www.ikp.kit.edu/corsika/.

[12] M. A. HAPGOOD, Planet. Space Sci., 40, 711-717 (1992). 